\title{
Capacitação da equipe de enfermagem sobre o reconhecimento precoce da deterioração do paciente hospitalizado
}

\author{
Nursing team training on early recognition of hospitalized patient deterioration
}

\begin{abstract}
RESUMO
Objetivo: Capacitar a equipe de enfermagem no reconhecimento precoce dos sinais de deterioração clínica do paciente hospitalizado. Métodos: Estudo intervencionista, com abordagem quantitativa, realizado em agosto e setembro de 2019 na equipe de enfermagem de quatro unidades de internação de um hospital escola no interior do estado de São Paulo, com aplicação do escore de alerta precoce modificado (Modified Early Warning Score - MEWS) e de questionário com 12 questões para avaliação pós-intervenção. Os resultados foram analisados por estatística descritiva. Resultados: A equipe de enfermagem é composta de 84 profissionais. Destes, foram capacitados 51 (60,7\%). A maioria soube identificar os sinais e sintomas de deterioração clínica de paciente hospitalizado (40; 78,4\%). O menor número de acertos foi em relação aos parâmetros que compõem a escala de MEWS (34; 66,6\%). Na Internação Médica/Cirúrgica do $3^{\circ}$ Leste e $2^{\circ}$ Oeste, o índice de acertos da equipe de enfermagem foi menor quando comparado ao dos demais setores. Conclusão: O estudo permitiu capacitar a equipe de enfermagem sobre a utilização do MEWS e a implantação do fluxograma de seguimento continuado do paciente. Embora dois setores tenham apresentado dificuldade em identificar os parâmetros que compõem a escala de MEWS e sua classificação, enfatiza-se a necessidade de capacitação contínua para que as medidas implementadas no fluxograma continuem tendo a eficácia desejada.

Palavras-chave: assistência hospitalar; parada cardíaca; educação em saúde; enfermagem.
\end{abstract}

\begin{abstract}
Objective: To train nursing staff to early recognize signs of clinical deterioration in hospitalized patients. Methods: Interventionist study with a quantitative approach, conducted in August and September of 2019 with nursing professionals from four hospitalization units of a hospital school located in the interior of the state of São Paulo, in which the Modified Early Warning Score (MEWS) and a questionnaire with 12 questions for post-intervention evaluation were applied. The results were analyzed by descriptive statistics. Results: The nursing team consisted of 84 professionals. Of these, $51(60.7 \%)$ were trained. Most were able to identify the signs and symptoms of clinical deterioration in hospitalized patients $(40 ; 78.4 \%)$. The lowest number of correct answers was about the parameters composing the MEWS scale (34; 66.6\%). In the Medical/Surgical Hospitalization Section of the 3rd East and 2nd West, the nursing team's correct answer rate was lower when compared to that of other sections. Conclusion: This study enabled nursing staff to be trained on the use of the MEWS and the implementation of the patient follow-up flowchart. Although two sectors had difficulty identifying the parameters that make up the MEWS scale and its classification, the need for continuous training is emphasized in order that the measures implemented in the flowchart will continue to have the desired effectiveness. Keywords: hospital care; heart arrest; health education; nursing.
\end{abstract}

'Pontifícia Universidade Católica de São Paulo, Faculdade de Ciências Médicas e da Saúde - Sorocaba (SP), Brasil. Autora correspondente: Izabel Cristina Ribeiro da Silva Saccomann - Pontifícia Universidade Católica de São Paulo, Faculdade de Ciências Médicas e da Saúde - Rua Joubert Wey, 290 - CEP: 18030-070 - Sorocaba (SP), Brasil E-mail: isaccomann@pucsp.br

Recebido em 03/12/2019. Aceito para publicação em 26/07/2021. 


\section{INTRODUÇÃO}

A última atualização das diretrizes da American Heart Association (AHA) sobre ressuscitação cardiopulmonar (RCP), ocorrida em 2015, acrescentou um novo elo à cadeia de sobrevivência para o ambiente intra-hospitalar. ${ }^{1}$ Esse elo visa à vigilância do paciente hospitalizado para evitar a parada cardiorrespiratória (PCR). Aproximadamente $80 \%$ dos sinais de deterioração clínica do paciente hospitalizado podem ser identificados 24 horas antes do agravamento do evento. ${ }^{2}$

Diariamente, são presenciados histórias de pacientes que apresentam quadro clínico estável, mas que evoluem com complicações, muitas vezes fatais. Medidas simples, de baixo impacto financeiro e grande eficácia poderiam mudar a evolução se fossem tomadas no momento certo. O não reconhecimento precoce dessas complicações levam à piora da doença, e, em muitos desses casos, mesmo em se adotando um conjunto de medidas complexas e de custo elevado, a resposta pode ser lenta e nem sempre resultar em sucesso. ${ }^{3}$ A equipe de enfermagem, por assistir o paciente durante 24 horas por dia, é a primeira a identificar uma possível PCR; assim, desempenha papel essencial no reconhecimento precoce dos sinais e sintomas de deterioração clínica do paciente.

Uma ferramenta para essa avaliação é o National Early Warning Score (NEWS), ou escore para alerta precoce (EPAP), desenvolvido pelo Royal College of Physicians $(\mathrm{RCP})^{4}$ para ser utilizado como instrumento de avaliação dos parâmetros fisiológicos que demonstram precocemente a deterioração clínica do paciente e que podem evoluir para uma PCR. Os hospitais que o adotam como ferramenta de diagnóstico viabilizam a realização de condutas que devem ser tomadas antecipadamente, visando à melhora do desfecho evolutivo e à diminuição dos riscos de agravo e contribuindo, também, para o menor custo de tratamento e assistência. ${ }^{5}$

Posteriormente, foi criada uma versão modificada, o escore de alerta precoce modificado (Modified Early Warning Score - MEWS), ${ }^{6}$ baseado na monitorização de parâmetros fisiológicos facilmente acessíveis: a pressão arterial sistólica, a frequência de pulso, a frequência respiratória, a temperatura e o nível de consciência. ${ }^{7}$ Um estudo que empregou o MEWS identificou maior gravidade no paciente com escore final superior a 5 pontos. ${ }^{8}$

A utilização do NEWS ou da versão modificada, MEWS, nas enfermarias objetiva a continuidade da assistência, permite melhor comunicação entre os membros da equipe de enfermagem, oferece autonomia profissional aos enfermeiros e melhora a relação enfermagem-médico, o que se repercute no melhor atendimento ao paciente. Por ser de ser de fácil aplicação, a ferramenta permite que, com base no escore obtido, seja possível selecionar os pacientes que necessitam de maior atenção da equipe de enfermagem. Um estudo demonstrou a eficácia dessa ferramenta, com redução significativa dos chamados para atendimentos em PCR, dada a identificação mais eficaz do paciente instável. ${ }^{9}$

Nesse contexto, a atualização e a capacitação relativas à nova diretriz da $\mathrm{AHA}$, com foco na vigilância e na pre- venção de danos à saúde do paciente, torna-se importante e indispensável para a assistência mais segura e qualificada. ${ }^{10}$ Os resultados de um estudo mostraram que a equipe de enfermagem reconhece o conjunto de sinais de piora clínica que pode gerar um evento de PCR $(86,5 \%)$; contudo, somente $43,6 \%$ dos profissionais conhecem a atualização das diretrizes da AHA e 22,6\% tinham conhecimento do novo elo da cadeia de sobrevivência. ${ }^{11}$ Assim, este estudo teve como objetivo capacitar a equipe de enfermagem quanto ao reconhecimento precoce dos sinais de deterioração clínica do paciente hospitalizado.

\section{MATERIAIS E MÉTODOS}

Trata-se de um estudo de natureza intervencionista e com abordagem quantitativa, realizado no Hospital Santa Lucinda (HSL), na cidade de Sorocaba. Foram incluídos os profissionais da equipe de enfermagem que atuam nas unidades de internação do hospital e que concordaram em participar do estudo por meio do Termo de Consentimento Livre e Esclarecido. Este trabalho foi aprovado pelo Comitê de Ética em Pesquisa (CEP) da Faculdade de Ciências Médicas e da Saúde da Pontifícia Universidade Católica (FCMS-PUC/SP), sob Parecer n ${ }^{\circ} 3.434 .779$, e está de acordo com a Resolução no 466/2012 do Conselho Nacional de Saúde e com as diretrizes e normas regulamentadoras de pesquisa envolvendo seres humanos. A coleta de dados foi aprovada pelo HSL. Foram excluídos os profissionais da equipe de enfermagem que se encontravam em férias ou licença médica no período do treinamento e aqueles que não aceitaram participar dele.

A capacitação foi realizada entre os meses de agosto e setembro de 2019, com a equipe de enfermagem de quatro unidades de internação: Internação Cirúrgica, $3^{\circ}$ andar, Leste e Oeste e Internação Clínica Médica/Cirúrgica, $2^{\circ}$ andar e andar térreo. Foi oferecida nos três turnos - manhã, tarde e noite -, em horários acordados com a Educação Continuada da instituição para não interferir na rotina de trabalho dos profissionais. A população total da pesquisa era de 84 profissionais que trabalhavam nos quatro setores, e a amostra do estudo foi do tipo aleatória simples, sem reposição, constituindo o total de 51 profissionais de enfermagem, entre enfermeiros, técnicos e auxiliares que atenderam aos critérios de inclusão e exclusão.

A intervenção do estudo constitui-se em demonstração e interpretação do MEWS para o reconhecimento precoce dos sinais de deterioração clínica do paciente, seguidas da aplicação do fluxograma no próprio setor de trabalho, com duração de aproximadamente $30 \mathrm{~min}$. Para a aplicabilidade da escala e a viabilização da assistência, o HSL optou pela padronização do fluxograma de atendimento utilizado pelo Hospital Vera Cruz da cidade de Campinas, que apresentou resultados positivos na identificação objetiva e precoce de sinais de deterioração clínica do paciente. ${ }^{12}$ Após a capacitação, aplicou-se um questionário elaborado pelas autoras do estudo, com 12 questões fechadas que avaliam o conhe- 
cimento adquirido após a intervenção, com vistas a avaliar a sua eficácia.

O MEWS é interpretado pela soma total dos escores ou pontos. A atribuição de pontos varia de zero a três para cada um dos parâmetros, indicando, assim, se há deterioração clínica. Após a avaliação de todas as variáveis, realiza-se a soma dos pontos para que seja definido o nível/grau do estado do paciente. Caso o resultado seja igual ou maior que 4 , imediatamente o médico da unidade deve ser informado para que o paciente receba os cuidados devidos em tempo hábil. ${ }^{2}$ Os dados revelados por essa escala são considerados objetivos na medida em que os diferentes valores dos sinais vitais aferidos são convertidos para escores que, após somados, traduzem diferentes graus de risco. Quanto mais distantes os parâmetros estiverem da normalidade, mais alta a pontuação. ${ }^{13}$

A interpretação dos dados foi feita por meio de análise descritiva e os resultados obtidos estão apresentados sob a forma de tabelas, em frequência absoluta e relativa.

\section{RESULTADOS}

A capacitação foi realizada nas quatro unidades de internação do HSL por se tratar de unidades que recebem pacientes no pré e pós-operatório, os quais podem apresentar sinais e sintomas de piora clínica. A equipe de enfermagem dessas unidades é composta de 84 funcionários. Destes, $51(60,7 \%)$ concordaram em participar da capacitação e $33(39,3 \%)$ foram excluídos - 14 estavam de folga ou haviam sido remanejados para outro setor, dois estavam em licença-maternidade, dois em licença médica, três de férias e 12 recusaram-se a participar. Entre os que aceitaram participar, $16(31,4 \%)$ eram da Internação Médica/Cirúrgica do $3^{\circ}$ andar Leste, $12(23,5 \%)$ da Internação Médica/Cirúrgica do $3^{\circ}$ andar Oeste, 14 (27,5\%) da Internação Clínica Médica/ Cirúrgica do $2^{\circ}$ andar e nove $(17,6 \%)$ da Internação Clínica Médica/Cirúrgica do térreo.

A Tabela 1 mostra o índice de acertos do questionário de avaliação do conhecimento adquirido após a capacitação.

Tabela 1. Acertos do questionário de avaliação do conhecimento adquirido após a capacitação. Sorocaba, 2019.

\begin{tabular}{|c|c|c|c|c|}
\hline \multirow{3}{*}{ Conhecimento } & \multirow{2}{*}{$\begin{array}{c}\text { Internação médica/ } \\
\text { cirúrgica } \\
3^{\circ} \text { Leste }\end{array}$} & \multirow{2}{*}{$\begin{array}{l}\text { Internação médica/ } \\
\text { cirúrgica } \\
3^{\circ} \text { Oeste }\end{array}$} & $\begin{array}{l}\text { Internação médica/ } \\
\text { cirúrgica }\end{array}$ & \multirow{2}{*}{$\begin{array}{c}\text { Internação médica/ } \\
\text { cirúrgica } \\
\text { Térreo }\end{array}$} \\
\hline & & & $2^{\circ}$ Oeste & \\
\hline & $\mathbf{n}=16(\%) * *$ & $\mathrm{n}=12(\%)$ & $n=14(\%)$ & $\mathbf{n}=\mathbf{9}(\%)$ \\
\hline $\begin{array}{l}\text { 1.Sinais de deterioração clínica de } \\
\text { paciente hospitalizado. }\end{array}$ & $13(25,5)$ & $12(23,5)$ & $8(15,7)$ & $7(13,7)$ \\
\hline 2.Finalidade da escala de MEWS. & $10(19,6)$ & $12(23,5)$ & $11(21,6)$ & $6(11,8)$ \\
\hline $\begin{array}{l}\text { 3.Parâmetros que compõem a escala } \\
\text { de MEWS. }\end{array}$ & $09(17,6)$ & $11(21,6)$ & $9(17,6)$ & $5(9,8)$ \\
\hline 4.Pontuação da escala de MEWS. & $13(25,5)$ & $12(23,5)$ & $13(25,5)$ & $9(17,6)$ \\
\hline $\begin{array}{l}\text { 5.Classificação correta do paciente } \\
\text { com escore até 2: baixo risco. }\end{array}$ & $12(23,5)$ & $11(21,6)$ & $11(21,6)$ & $8(15,7)$ \\
\hline $\begin{array}{l}\text { 6.Classificação correta do paciente } \\
\text { com escore entre } 3 \text { e } 4 \text { : } \\
\text { risco moderado. }\end{array}$ & $10(19,6)$ & $12(23,5)$ & $13(25,5)$ & $7(13,7)$ \\
\hline $\begin{array}{l}\text { 7.Classificação correta do paciente } \\
\text { com escore 5: alto risco. }\end{array}$ & $15(29,4)$ & $12(23,5)$ & $13(25,5)$ & $8(15,7)$ \\
\hline $\begin{array}{l}\text { 8. Conduta correta com seguimento } \\
\text { do fluxograma para pacientes com } \\
\text { escore } 0 \text { ou } 1 \text {. }\end{array}$ & $15(29,4)$ & $12(23,5)$ & $13(25,5)$ & $9(17,6)$ \\
\hline $\begin{array}{l}\text { 9. Conduta correta com seguimento } \\
\text { do fluxograma para pacientes com } \\
\text { escore } 2 \text {. }\end{array}$ & $11(21,6)$ & $09(17,6)$ & $10(19,6)$ & $7(13,7)$ \\
\hline $\begin{array}{l}\text { 10. Conduta correta com seguimento } \\
\text { do fluxograma para pacientes com } \\
\text { escore } 3 \text { e } 4 \text {. }\end{array}$ & $14(27,5)$ & $12(23,5)$ & $13(25,5)$ & $7(13,7)$ \\
\hline $\begin{array}{l}\text { 11. Conduta correta com seguimento } \\
\text { do fluxograma para pacientes com } \\
\text { escore } 5 \text {. }\end{array}$ & $14(27,5)$ & $11(21,6)$ & $13(25,5)$ & $8(15,7)$ \\
\hline $\begin{array}{l}\text { 12.Compreenderam o objetivo da } \\
\text { utilização do MEWS nas enfermarias }\end{array}$ & $10(19,6)$ & $12(23,5)$ & $14(27,5)$ & $6(11,8)$ \\
\hline
\end{tabular}

Intern. Med/Cirur: internação médica/cirúrgica; MEWS: escore de alerta precoce modificado. 
A maioria $(40 ; 78,4 \%)$ soube descrever os sinais e sintomas de deterioração clínica do paciente hospitalizado. O menor número de acertos $(34 ; 66,6 \%)$ foi em relação aos parâmetros que compõem a escala de MEWS. Na Internação Médica/Cirúrgica do $3^{\circ}$ Leste e $2^{\circ}$ Oeste, o índice de acertos da equipe de enfermagem foi menor quando comparado ao dos demais setores.

\section{DISCUSSÃO}

A capacitação realizada mostrou que equipe de enfermagem soube descrever os sinais e sintomas precoces da deterioração clínica do paciente. Estudos indicam que alterações de sinais fisiológicos precedem em horas a situação de deterioração do estado clínico. ${ }^{14}$ Por isso, a identificação precoce de pacientes com risco de deterioração clínica, combinado à gravidade da doença, é um componente integral da assistência médica de alta qualidade. ${ }^{15}$ Entretanto, alguns estudos demonstram que os enfermeiros e sua equipe apresentam um desconhecimento significativo em relação à identificação de um paciente em PCR e à sequência atual para o suporte básico de vida. ${ }^{16,17}$

O pior desempenho foi para a questão que avalia os parâmetros que compõem a escala de MEWS. Esta, baseada em parâmetros fisiológicos, permite identificar precocemente níveis de deterioração clínica do paciente ${ }^{13}$ e consequentemente agir com maior prontidão por meio de um atendimento rápido e seguro diante da piora clínica do paciente. Vale ressaltar que os parâmetros que compõem os escores dessa escala definirão a permanência ou não do paciente no setor de internação. Um estudo realizado nas alas de internação de um hospital demonstrou que, após a implantação do protocolo com a utilização da escala de MEWS, houve redução do número de transferências desnecessárias dos pacientes para a unidade de terapia intensiva, permanecendo $93 \%$ deles no setor de internação. ${ }^{12}$

Uma forma de superar essa fragilidade é a capacitação contínua da equipe de enfermagem, cuja importância foi evidenciada pelo baixo índice das respostas sobre a utilização do MEWS em duas unidades. Um estudo revelou que após o treinamento dos profissionais na implantação do MEWS houve melhora nos registros de enfermagem do prontuário dos pacientes. Sendo assim, o conhecimento científico e a prestação de assistência qualificada dão-se por meio de capacitações periódicas obtidas em treinamentos frequentes no ambiente de trabalho e proporcionadas pela própria instituição. ${ }^{18}$

A equipe de enfermagem soube definir corretamente as pontuações do escore da escala MEWS que classificam o paciente conforme o baixo, moderado ou alto risco de deterioração clínica. Entretanto, o setor de internação $3^{\circ}$ Leste teve uma pontuação abaixo do esperado na classificação dos escores 3 e 4, considerado como risco moderado. Esse desconhecimento pode desencadear algumas situações que irão interferir no acompanhamento do paciente. De acordo com o fluxograma, os escores 3 ou 4 devem ser informados ao enfermeiro e ao médico, e o paciente reavaliado a cada 2 horas. Assim, uma classificação realizada de forma inapropriada pode subestimar uma alteração dos sinais e sintomas que permita avaliar a piora clínica do paciente, com consequente diminuição das chamadas de alerta de PCR. As evidências apontam forte associação entre a presença de escores críticos (> 3 pontos) e a deterioração clínica dos pacientes cujos parâmetros já se achavam alterados 72 horas antes da admissão, ${ }^{7}$ revelando assim a importância da vigilância precoce. Outros estudos também demonstraram que os desfechos (óbito, transferência para centro de terapia intensiva) foram significativamente maiores no grupo avaliado pelo MEWS. ${ }^{2}$

A literatura revela que os resultados do protocolo do MEWS são necessários para se conhecer a realidade do setor, identificar as fragilidades e propor melhorias. Um estudo realizado em um hospital da Noruega com enfermeiros mostrou que, após um programa de educação sobre o MEWS, houve melhoria na detecção de mudanças no quadro do paciente, auxiliando na tomada de decisões clínicas. ${ }^{19}$ Outro estudo apontou aumento do percentual de estabilização do paciente na unidade, indicando detecção precoce da deterioração clínica, com resolução mais rápida. ${ }^{18} \mathrm{O}$ direcionamento de medidas precoces e mais intensivas para evitar a piora clínica do paciente tem-se mostrado eficiente uma vez que há relação direta entre a presença de um escore de alto risco e a crescente morbimortalidade. ${ }^{2}$

Outro ponto importante a ser considerado é que essa escala permite melhor comunicação, oferece autonomia profissional aos enfermeiros e melhora a relação enfermagem-médico, o que se repercute no melhor atendimento ao paciente, além de ser considerada uma ferramenta de segurança no cuidado. ${ }^{20} \mathrm{~A}$ Meta Internacional de Segurança do Paciente preconiza a melhoria da comunicação entre os profissionais da saúde, garantindo que as informações verbais referentes aos pacientes sejam precisas e completas, bem como a forma do seu registro. ${ }^{21}$ Entretanto, um estudo revela a dificuldade de comunicação das alterações dos sinais e sintomas pelos técnicos de enfermagem. ${ }^{22} \mathrm{O}$ fluxo de atendimento e o processo de comunicação deficientes entre os profissionais acarretam atrasos na resolução dos problemas, descontentamento dos pacientes e desfechos clínicos indesejáveis, pois a tomada de decisão perante a deterioração clinica interfere diretamente no resultado final do atendimento. ${ }^{23}$

Por fim, durante toda a capacitação, perceberam-se o comprometimento da maioria dos profissionais com a melhoria da assistência e a conscientização sobre a importância da detecção precoce de sinais e sintomas de piora clínica dos pacientes da enfermaria. Experiência semelhante foi relata por Pereira et al. ${ }^{14}$ durante a implantação de escore de alerta de gravidade precoce em um hospital infantil, que possibilitou aos enfermeiros maior segurança no gerenciamento do tempo de trabalho e aos técnicos a visualização imediata dos intervalos de normalidade dos sinais vitais, favorecendo a solicitação adequada de suporte do enfermeiro. 


\section{CONCLUSÕES}

O estudo permitiu capacitar a equipe de enfermagem das unidades de internação do HSL sobre a utilização do MEWS como ferramenta clínica capaz de detectar precocemente os sinais de deterioração clínica. O MEWS é um instrumento simples aplicada à beira do leito e pode ser interpretada pela equipe de enfermagem na tentativa de identificar os pacientes de alto risco. Além disso, com a implantação do fluxograma, foi possível orientar o seguimento continuado dos pacientes, a definição dos que necessitam de maior atenção da equipe responsável e o encaminhamento daqueles com os escores alterados, posto que há evidências de que a intervenção precoce pode melhorar o desfecho evolutivo.

Este novo protocolo da instituição foi muito bem recebido pela equipe de enfermagem, que acreditou na possibilidade de melhora na qualidade e segurança do serviço oferecido. Como todo processo novo que é implantado, encontramos algumas dificuldades no início, principalmente pelo fato de o treinamento ter sido realizado in loco, com algumas interrupções. $\mathrm{O}$ grande desafio foi conscientizar a equipe sobre a importância dessa ferramenta para a melhora da qualidade da assistência prestada, assim como para a segurança do paciente hospitalizado.

Visto que dois setores apresentaram dificuldade em identificar os parâmetros que compõem a escala de MEWS e sua classificação, enfatiza-se a necessidade de capacitação contínua para que as medidas implementadas no fluxograma continuem tendo a eficácia desejada.

\section{REFERÊNCIAS}

1. American Heart Association. Destaques das Diretrizes da American Heart Association 2015 para RCP e ACE. Dallas: American Heart Association; 2015.

2. Rocha TF, Neves JG, Viegas K. Escore de alerta precoce modificado: avaliação de pacientes traumáticos. Rev Bras Enferm. 2016;69(5):906-11. https://doi. org/10.1590/0034-7167-2015-0145

3. Ludikhuize J, Smorenburg SM, Rooij SE, Jonge E. Identification of deteriorating patients on general Ward measurement of vital paremeters and potential effectivenes of the Moified Early Warnig Score. J Crit Care. 2012;27(4):424.e7-13. https://doi. org $/ 10.1016 /$ j.jcrc.2012.01.003

4. Royal College of Physicians. National Early Warning Score (NEWS): Standardising the assessment of acute illness severity in the NHS. Report of a working party. Londres: RCP; 2012.

5. Hospital Universitário da Universidade Federal de Juiz de Fora (HU-UFJF). Escore para alerta precoce [Internet]. 2019 [acessado em 10 nov. 2019]. Disponível em: http://www2.ebserh.gov.br/web/huufjf/escore-para-alerta-precoce

6. Kyriacos U, Jelsma J, Jordan S. Record review to explore the adequacy of post-operative vital signs monitoring using a local Modified Early Warning Score (Mews) Chart to Evaluate Outcomes. PLoS One. 2014;9(1):e87320. https://doi.org/10.1371/ journal.pone. 0087320

7. Tavares RCF, Vieira AS, Uchoa LV, Junior AAP, Meneses FA. Validação de um escore de alerta precoce pré-admissão na unidade de terapia intensiva. Rev Bras Ter Intensiva. 2008;20(2):124-7. https://doi. org/10.1590/S0103-507X2008000200002

8. Subbe CP, Kruger M, Rutherford P, Gemmel L. Validation of a modified Early Warning Score in medical admissions. QJM. 2001;94(10):521-6. https://doi.org/10.1093/qjmed/94.10.521

9. Fullerton JN, Price CL, Silvey NE, Brace SJ, Perkins GD. Is the Modified Early Warning Score (MEWS) superior to clinician judgement in detecting critical illness in the pre-hospital environment? Resuscitation. 2012;83(5):557-62. https://doi. org/10.1016/j.resuscitation.2012.01.004

10. Puggina CC, Amestoy SC, Fernandes HN, Carvalho LA, Báo ACP, Alves F. Educação permanente em saúde: instrumento de transformação do trabalho de enfermeiros. Espaç Saúde. 2015;16(4):87-97. https://doi.org/10.22421/15177130-2015v16n4p87

11. Jacintho P, Aparecida MC. Conhecimento da equipe de enfermagem sobre vigilância e prevenção do paciente hospitalizado. In: Anais do $28^{\circ}$ Encontro de Iniciação Científica da PUC/SP. São Paulo: PUCSP; 2019.

12. Santos FF, Bueno S. Resultados da Implantação do Protocolo Código Amarelo nas alas de internação [Internet]. 2016 [acessado em 10 nov. 2019]. Disponível em: http://www.hospitalveracruz. com.br/varal-cientifico/assets/pdf/poster-codigoamarelo

13. Mendes TJM, Silveira LM, Silva LP, Stabile AM. Associação entre o acolhimento com classificação de risco, desfecho clínico e o escore MEWS. REME. 2018;22(e-1077):1-6. http://www.dx.doi. org/10.5935/1415-2762.20180007

14. Pereira R, Mansur DGN, Ionemoto HF. Implantação de escore de alerta de gravidade precoce em Hospital Infantil privado: relato de experiência. Rev Soc Bras Enferm Ped. 2016;16(2):81-4. http://dx.doi. org/10.31508/1676-3793201600011

15. Gold DL, Mihalov LK, Cohen DM. Evaluating the Pediatric Early Warning Score (PEWS) system for admitted patients in the pediatric emergency department. Acad Emerg Med. 2014;21(11);124956. https://doi.org/10.1111/acem.12514

16. Almeida AO, Araujo IEM, Darli MCB, Araujo S. Theoretical knowledge of nurses working in nonhospital urgent and emergency care units concerning cardiopulmonary arrest and resuscitation. Rev Latinoam Enferm. 2011;19(2):261-8. https://doi. org/10.1590/S0104-11692011000200006 
17. Rocha FAZ, Oliveira MCL, Cavalcante RB, Silva PC, Rates HF. Atuação da equipe de enfermagem frente à parada cardiorrespiratória intrahospitalar. $\mathrm{R}$ Enferm Cent O Min. 2012;2(1):141-50. https://doi. org/10.19175/recom.v0i0.100

18. Cipriano ESV, Salgado BS, Oliveira NA, Aguiar BGC. Implantação do Score de deterioração clínica (MEWS) em um hospital privado da cidade do Rio de Janeiro e seus respectivos resultados. Enferm Brasil. 2018;17(1):3442. https://doi.org/10.33233/eb.v17i1.2241

19. Stafseth SK, Gronbeck S, Lien T, Randen I, Lerdal A. The experiences of nurses implementing the Modified Early Warning Score and a 24-hour on-call Mobile Intensive Care Nurse: an exploratory study. Intens Crit Care Nurs. 2016;34:33-41. https://doi.org/10.1016/j. iccn.2015.07.008
20. Olino L, Gonçalves AC, Strada JKR, Vieira LB, Machado MLP, Molina KL, et al. Comunicação efetiva para a segurança do paciente: nota de transferência e Modified Early Warning Score. Rev GaúchaEnferm. 2019;40(spe.):19. https://doi.org/10.1590/1983-1447.2019.20180341

21. Agência Nacional de Vigilância Sanitária. Assistência segura: uma reflexão teórica aplicada à prática. Brasília: Anvisa; 2017.

22. Paula KO, Reis LKBB, Costa SM, Fernandes GCG. Conhecimento e conduta da equipe de Enfermagem frente aos sinais e sintomas que precedem a parada cardiorrespiratória na clínica médica cirúrgica. Ensaios USF. 2018;2(1):1-11.

23. XVIII Encontro Latino-Americano de Iniciação Científica, XIV Encontro Latino-Americano de Pósgraduação e IV Encontro de Iniciação à Docência; 23-24 out. 2014. Anais. Universidade do Vale do Paraíba; 2014.

\section{Como citar este artigo:}

Jacintho P, Aparecida MC, Saccomann ICRS. Capacitação da equipe de enfermagem sobre o reconhecimento precoce da deterioração do paciente hospitalizado. Rev Fac Ciênc Méd Sorocaba. 2020;22(3):119-24. https://doi.org/10.23925/19844840.2020v22i3a6 